\title{
Research Paper \\ Forecasting Suicide Based on Sexuality, Marital Status, Coping Strategies, Religious Orientation, and Depression Rate
}

\author{
${ }^{*}$ Yasaman Emad ${ }^{1}(\mathbb{1}$, Habib Hadianfard² $(1)$
}

1. MA. in Clinical Psychology, Department of Clinical Psychology, School of Education \& Psychology, Shiraz University, Shiraz, Iran. 2. PhD. in Clinical Psychology, Department of Clinical Psychology, School of Education \& Psychology, Shiraz University, Shiraz, Iran.

\begin{tabular}{|c|c|}
\hline $\begin{array}{l}\text { Use your device to scan } \\
\text { and read the article online }\end{array}$ & dtration Emad Y, Hadianfard H. [Forecasting Suicide Based on Sexuality, Marital Status, Coping Strategies, Religious Orien- \\
\hline 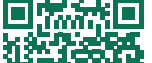 & $\begin{array}{l}\text { tation, and Depression Rate (Persian)]. Iranian Journal of Psychiatry and Clinical Psychology. 2019; 25(2):178-193. http://dx.doi. } \\
\text { org/10.32598/ijpcp.25.2.178 }\end{array}$ \\
\hline 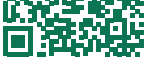 & doil http://dx.doi.org/10.32598/ijpcp.25.2.178 \\
\hline
\end{tabular}

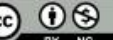

Received: 25 May 2018 Accepted: 25 Feb 2019 Available Online: $01 \mathrm{Jul} 2019$

Key words: Suicide risk, Gender, Marital status, Coping strategies, Religious orientation, Depression

\section{ABSTRACT}

Objectives The present study aimed at exploring the role of gender, marital status, coping strategies, religious orientation, and depression level in predicting suicidal tendencies using a correlational, and in some cases, practical methodology.

Methods The sample group included 260 bachelor students ( 175 male and 85 female; 213 single and 72 married) of Shiraz University in the academic year 2011-2012. The subjects were selected by a multi-stage cluster sampling method and then measured using Carver and Scheier Coping Strategy questioners (short form), Allport religious orientation questioners (short form), Beck Depression Inventory-II (BDI-II) and Beck Scale for Suicide Ideation (BSSI). The data were analyzed using enter regression method and SPSS 20 software.

Results Regression coefficients indicated that masculinity ( $B=0.83)$, ineffective coping style $(B=5.91)$, specifically coping strategies for denial $(B=1.04)$, self-blaming $(B=0.81)$ and non-behavioral conflict $(B=0.49)$ can positively and significantly predict the risk of suicide, while acceptance as coping strategy $(B=-0.48)$ predicts this risk negatively and significantly.

Conclusion The results showed that the above variables in the strongest predictive equation had $78 \%$ of the variance of suicide risk. Thus, when encountering the individuals exposed to suicidal risks, psychologists are expected to focus more on training coping methods.

\section{Extended Abstract}

\section{Introduction}

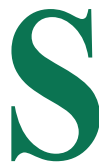

uicide is one of the serious public health concerns and the rate of suicidal thoughts is considered as one of the most important indicators of mental health through the communities [1]. In this regard, introducing some psychiatric disorders such as depression should be considered as the underlying reasons to com- mit suicide [5]. Depression, commonly referred to as the "common cold" of mental illness [6], can reduce the person's tolerance threshold and his ability to inhibit impulses, and ultimately increase the likelihood of committing suicide in the patients [7]. Several studies are conducted on the cognitive constructs involved in semiotics of suicide attempts that coping skills are one of the cognitive areas that can be considered. Carver and Scheier introduced three types of coping strategies. Emotion-focused coping includes seeking social support for emotional reasons, positive reinterpretation, acceptance, self-con-

* Corresponding Author:

Yasaman Emad, MA.

Address: Department of Clinical Psychology, School of Education \& Psychology, Shiraz University, Shiraz, Iran.

Tel: +98 (71) 36487417

E-mail: yasaman.emad@gmail.com 
trol, turning to the religion, and the emotional evacuation. Problem-focused coping includes social support as a tool, active coping, planning, and preventing disturbing activities. Finally, ineffective coping involves denial and mental or behavioral engagement with substances or alcohol indicating avoidance [36]. Both emotionalfocused coping and problem-oriented coping strategies can effectively reduce stress and tension-related problems [14]. Besides, other factors are involved such as religious orientation, which significantly can affect the risk of committing suicide [15]. According to Allport, there are two types of religious orientations. In individuals with an internal religious orientation who have internalized their accepted religion [16], and people with external religious orientation, the religion is emphasized due to its achievements [17].

Gender and marital status are studied in committing suicide. The female gender [19-24] and being married [1, 20, $23,25]$ are introduced by most studies as the risk factors to commit suicide. It is noteworthy that the male gender is described as a risk factor for committing suicide in some studies [5]. This contrast may be resolved by considering the effect of depression; accordingly, the female gender can increase the risk for depression due to the negative emotional-focused skills [16, 26]. Therefore, an increase in the likelihood of committing suicide [27] is practically due to the depression and ineffective coping strategies of the individual not because of his gender. The current study aimed at identifying the students at the risk of committing suicide more accurately along with the preventive measures considering the results of studies on risk factors in committing suicide and determining the predictive value of each of these factors.

\section{Method}

The present fundamental and correlational-descriptive study was conducted on all undergraduate students of the Shiraz University in 2011-12, of which 260 students [175 male and 85 female) were selected as the sample group. It should be noted that the number of sample group was calculated and determined based on the Cochran's formula. The age range of the subjects was 18 to 27 years old and their average age was 21 years. Among the studied students, 213 subjects were single and 72 married. Multistage cluster sampling was used as the sampling method. The inclusion criteria were age range 18 to 30 years old, being Iranian and Muslim, being literate, and willingness to participate in the study. The lack of psychiatric disorders such as mood and psychotic disorders or the disorders associated with drug abuse were also regarded. In the present study, four questionnaires were used to collect data including the Religious
Orientation Scale (ROS) developed by Allport, Short Form of the Carver and Scheier Coping Questionnaire, Beck Depression Questionnaire-II, and Beck Scale for Suicidal Ideation (BSSI). The questionnaires were scored and the subjects' scores were calculated, followed by using statistical techniques for data arrangement and summarization. To analyze the data, descriptive statistics indices were first determined and the regression method (entry method) was used in order to answer the hypothesis.

\section{Results}

In the current study, problem-focused and emotion-focused coping strategies were negatively and significantly correlated with the risk of committing suicide, and as assumed, the ineffective coping strategy had a positive and significant relationship with the risk of committing suicide. Meanwhile, among the three main types of coping strategies, the ineffective coping strategy had a predictive value.

According to the findings of the current study, among the fourteen-point coping strategies of Carver and Scheier, all strategies related to the ineffective coping strategy (self-confusion, denial, drug abuse, no behavioral involvement, emotional evacuation, and self-blaming) had a positive and significant correlation with the risk of committing suicide. However, there was a negative and significant correlation between the emotion-focused coping strategies (acceptance, humor, religion, positive framework, and emotional support) and the problemoriented coping strategies (planning, active coping, and tool support). The results indicated that ineffective coping strategy, denial, self-blaming, acceptance, no behavioral involvement, and gender can significantly explain $78 \%$ of the variance in suicide risk in an equation.

\section{Discussion}

According to the current study results, it seems that using the ineffective strategies to cope with the individuals' problems can lead to some disorders such as depression and suicide. In this regard, Cole's psychological stresspreparedness model provides a framework to treat people committing suicide [49]. Based on this model, subjects with difficulty in divergent thinking ability are not prepared to develop alternative solutions to deal effectively with such conditions, while they are normally under stressful situations. Accordingly, these people are disappointed and may find suicide as the only available solution, therefore, it seems that it is more related to the problem-solving and coping strategies of the individual. 
To explain the positive and significant relationship between depression and suicidal thoughts in the current study, there are several theoretical supports correlating depression-related cognitive function to suicidal thoughts and behavior and introducing suicide as an associate or the outcome of depression [55]. Although religious orientation in the current study was not a predictive value, due to the reverse and significant relationship between religion and suicidal thoughts, it can be concluded that religion, due to the obvious prohibition of suicide, and spiritual and religious beliefs to accept miserable events, adaptive behavior, patience against unpleasant incidents and having a positive attitude along with self-esteem to yourself and the future, and avoiding disappointment have an important role to diminish the psychosocial factors of depression and consequently committing suicide. In addition, according to the current study findings, the male gender can significantly predict the risk of suicide. To justify the results of the current study , it can be referred to the Parchi's view [63]. He believed that males have more impulsivity than females due to the increased levels of testosterone during maturity. It is not surprising to consider the male gender as one of the risk factors for suicide, since suicide is a self-destructive impulse, therefore, under similar circumstances, males are more likely to commit suicide [63].

To explain other findings, it can be implicitly assumed that early marriage (18-25 years old) can put a significant psychological pressure on couples due to the various concerns such as unemployment, financial problems, etc. Committing suicide may be the only solution available if couples do not use effective strategies to solve their problems; since stresses become persistent.

\section{Ethical Considerations}

\section{Compliance with ethical guidelines}

The study protocol was approved by University of Shiraz Ethics Committee, Iran. Informed consent was obtained from all the participants. Patients' right to withdraw from the study at any stage and the confidentiality of their responses was emphasized.

\section{Funding}

This study is extracted from the first author's master thesis at Shiraz University. The study was of no cost to the departmental staff. The study did also not impact Shiraz University's resources.

\section{Authors contributions}

Conceiving and designing the study: All authors; Providing data, drafting the paper: Yasaman Emad; Analyzing the data: All authors. Revising the work: Habib Hadianfard.

\section{Conflicts of interest}

The authors declared no conflict of interest. 


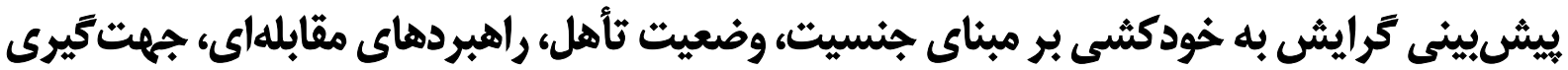 مذهبى و ميزان افسردئى كئس
}

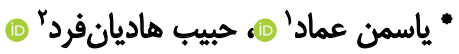 \\ 1- كارشناس ارشد روانشناسى بالينى، كروه روانشناسى بالينى، دانشكده علوم تربيتى و روانشناسى، دانشكاه شيراز، شيرازء، ايران.

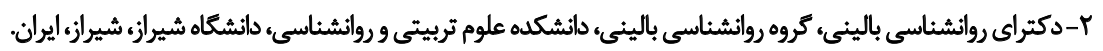

\begin{abstract}
حكيد

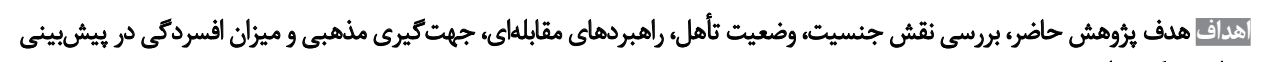
خطر خودكثى است.

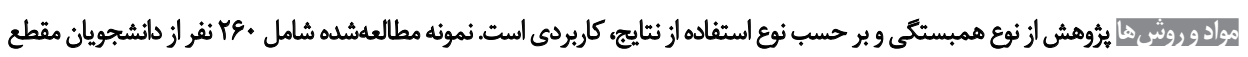

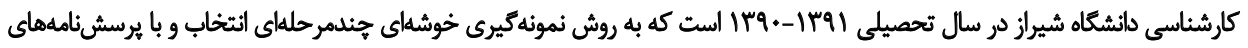

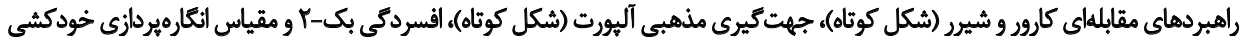

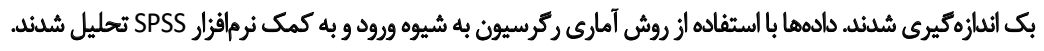

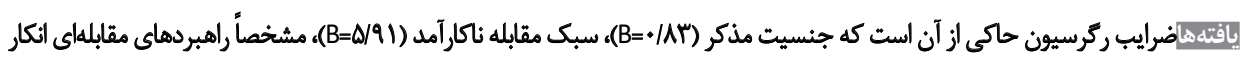

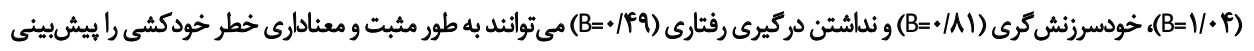

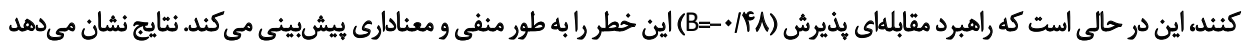

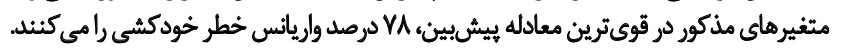

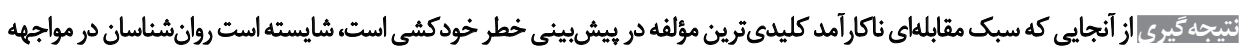

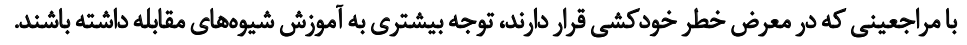

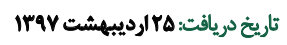

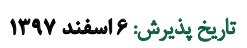

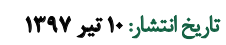

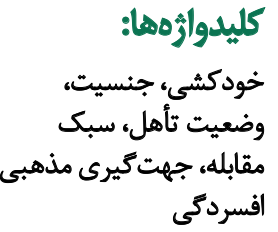

افسردكى جماني
اجتماعى ثلقى ميشد و در تبيين آن، عمدتاً متغيرهاى

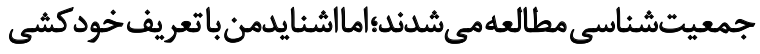

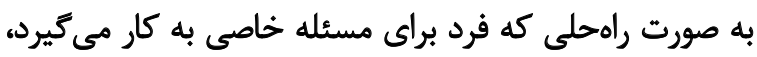

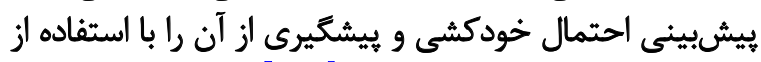

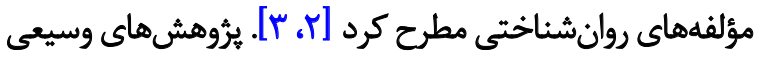

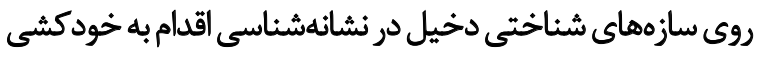

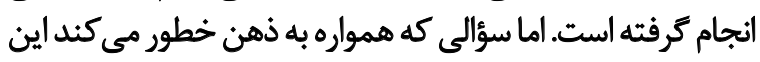

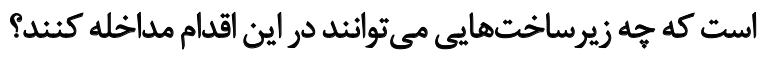

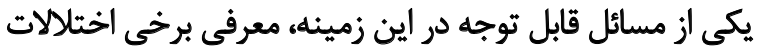

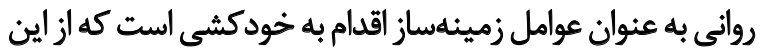
ميان مى توان به افسردگى اشاره كرد [F].

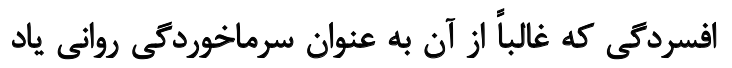

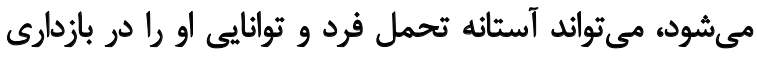

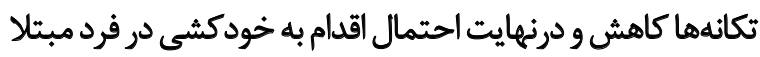

مقدمه خودكشى' يكى از مشكلات جدى سلامت عمومى بـى است و

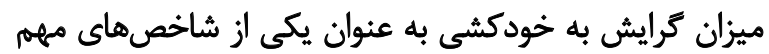

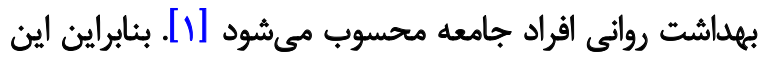

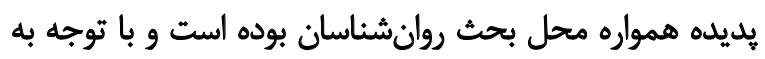

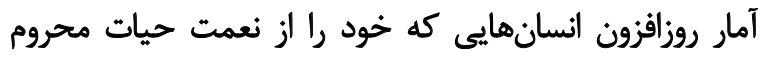

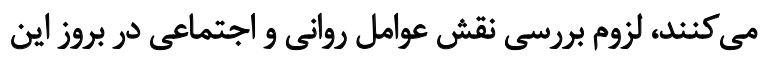

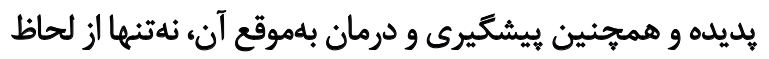

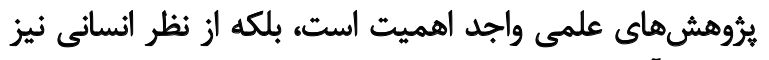
مى توان آن رانوعى وظيفه تلقى كرد.

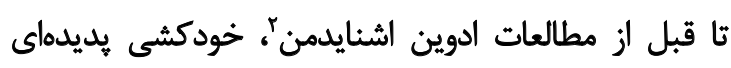

1. Suicide

2. Schneidman

* نويسئده مسئول: ياسمن عماد نشانى: شيراز، دانشكاه شيراز، دانشكده علوم تربيتى و روانشناسى، كروه روانشئاسى بالينى.

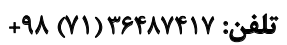
يست الكترونيكى: بلفي yasaman.emad@gmail.com 
مقابله مسئلهمدار، جستوجوى حمايت اجتماعى بادلايل ابزارى،

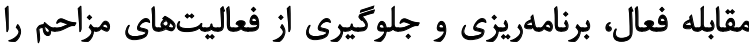

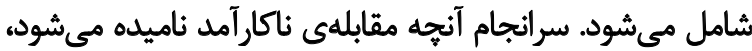

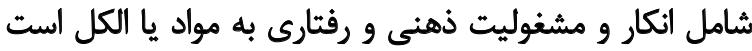

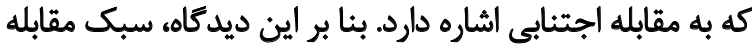

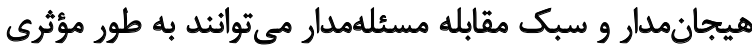

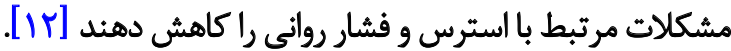

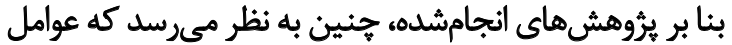

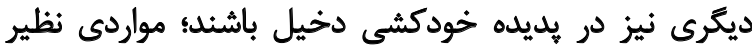

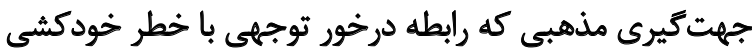

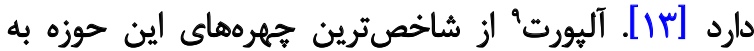

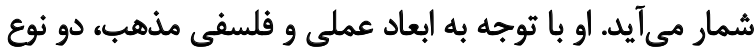

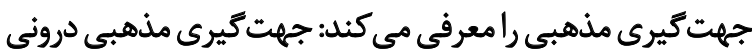

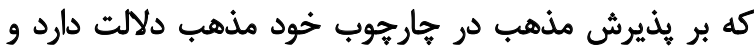

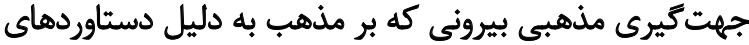

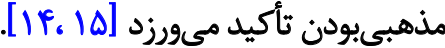
به زعم آليورت، كسانى كه مذهب بيرونى دارند، از نظر فلسفه

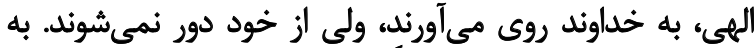

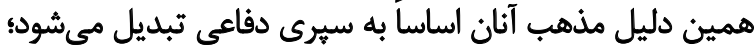

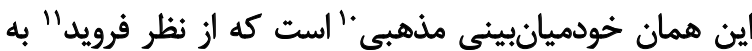

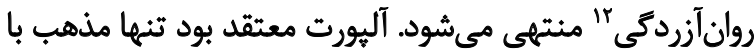

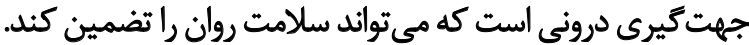

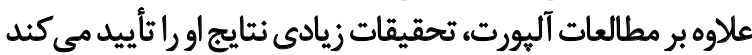

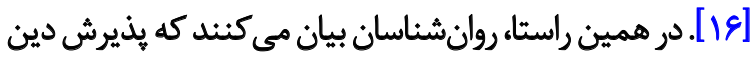

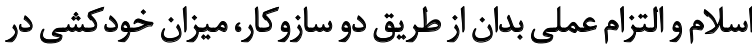

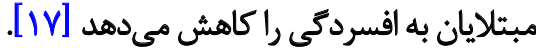

متغيرهاى ديكرى كه در اقدام به خودكشى بررسى شدهانده

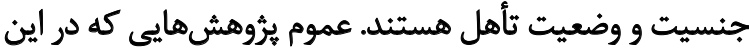

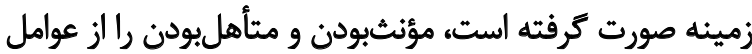

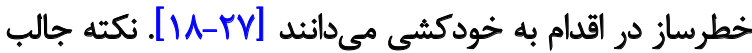

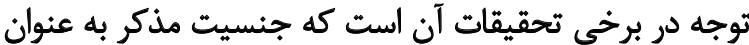

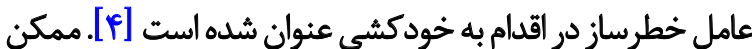

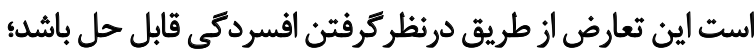

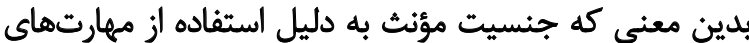

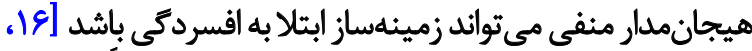

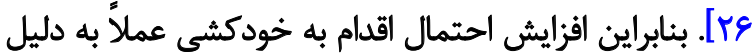

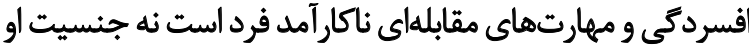

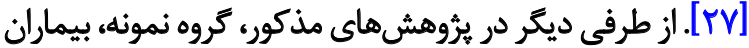

\section{Alport}

10. Religious Ego-centeric

11. Freud

12. Neurosis

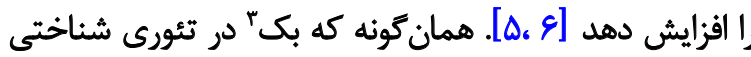

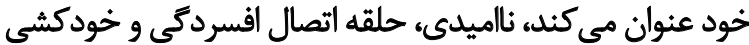

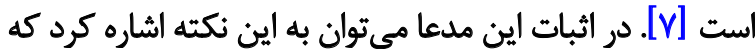

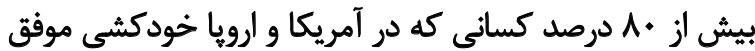

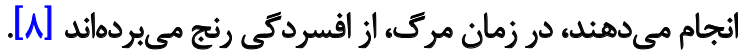
يكى ديكر از حوزههاى شناختى كه در اين راستا مىتواند

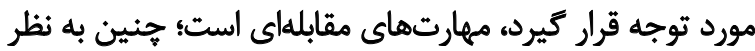

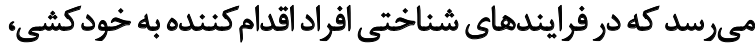

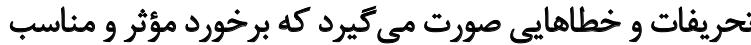

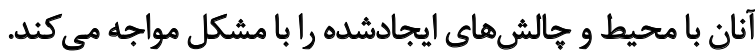

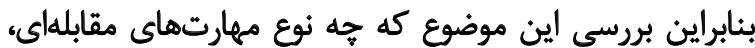

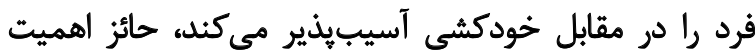

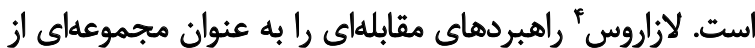

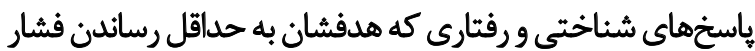

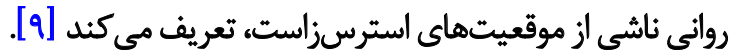
او در ثئورى خود به دو نوع سبك مقابلهاى اشاره مى كند:

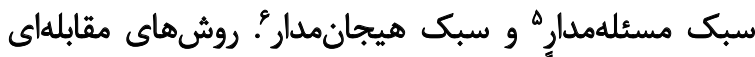

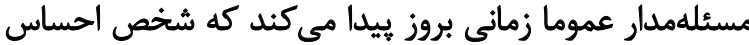

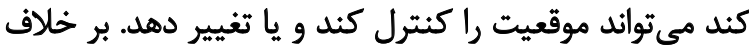

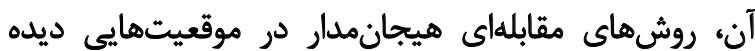

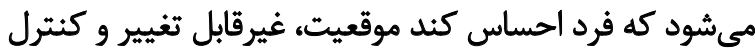

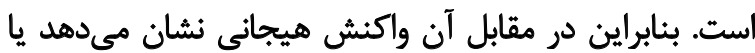
سعى در تحمل آن دارد [.1] فئ.

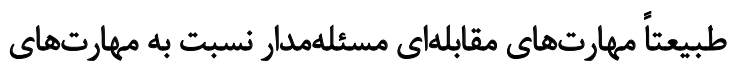

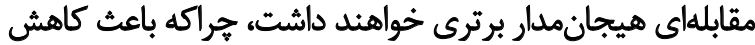

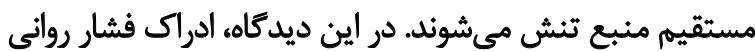

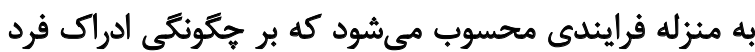

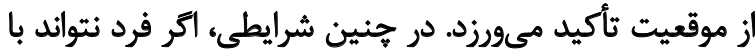

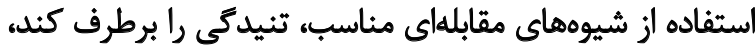

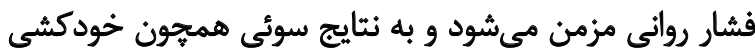

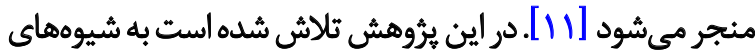

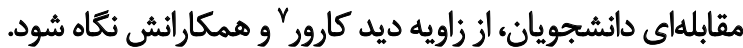
كارور و شيرر^ در سال 1919 سه سبك مقابله را معرفى

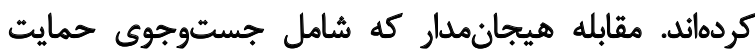

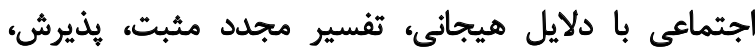
خويشتندارى، روىآوردن به مذهب ولاني تفير مجليه هيجانى است.

3. Beck

4. Lazarus

5. Problem-focused

6. Emotion-focused

7. Carver

8. Scheier 


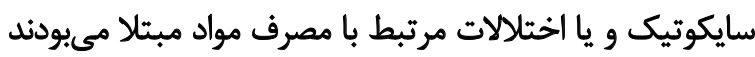

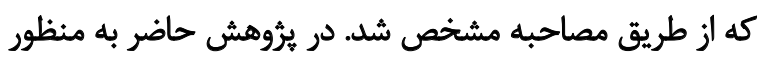

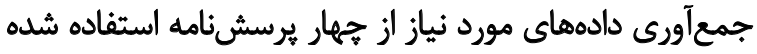

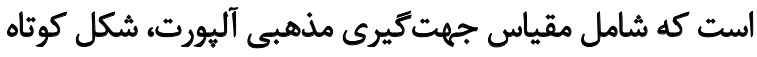

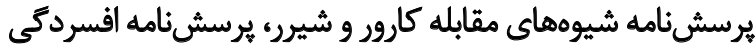

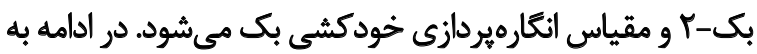

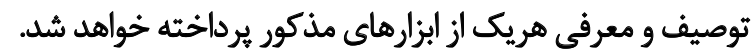

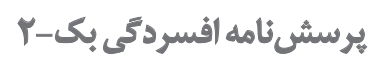

ويرايش دوم برسشنامه افسردكى بك كه در سال 1999

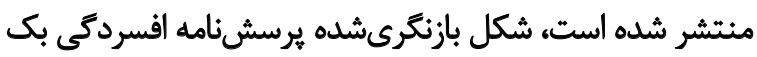

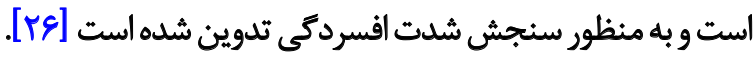

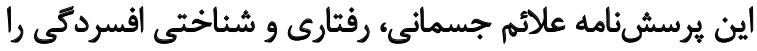

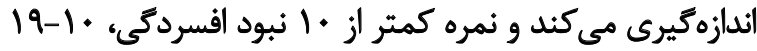

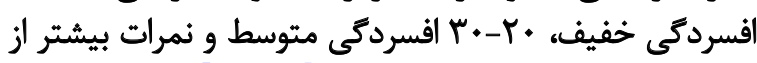

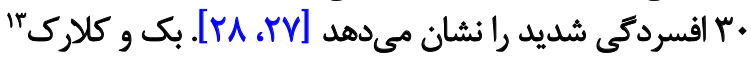

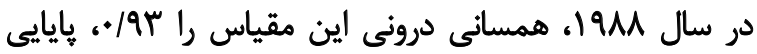

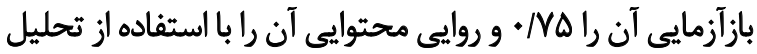

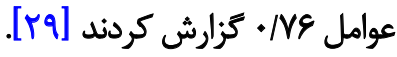

ترجمه و هنجاريابى اين يرسشنامه رائ را براى نخستينبار در

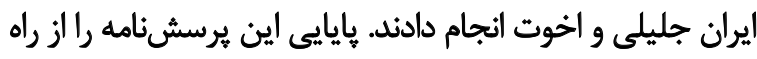

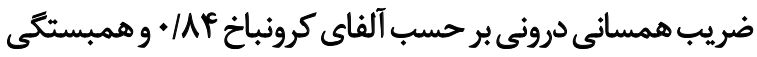

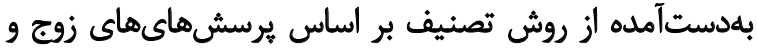

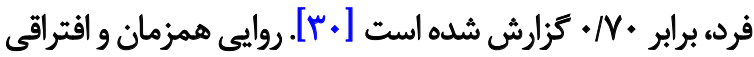

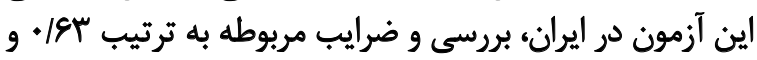

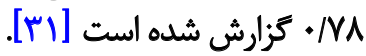

مثياس جهت مئيوى مذهبى آليورت (شكل كوتاه) (197V) اين يرسشنامه، شامل 10 ماده است كه 9 ماده اول،

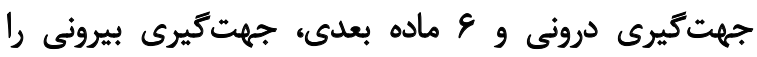

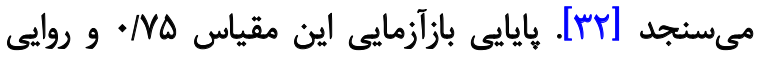

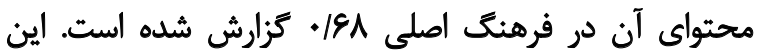

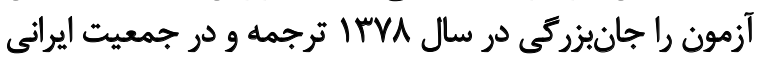

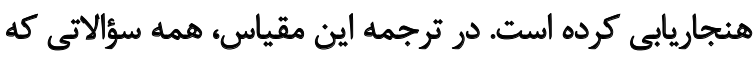

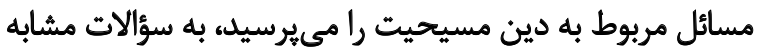

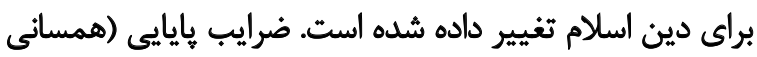

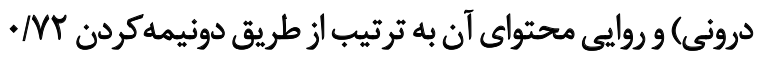

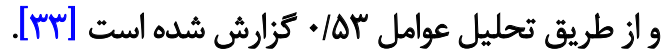

13. Clark

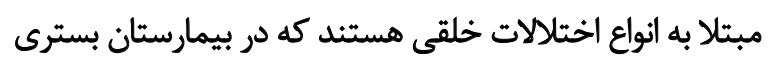

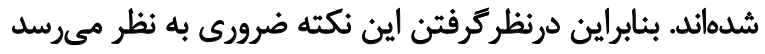

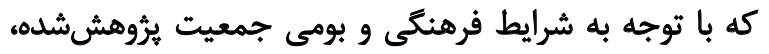

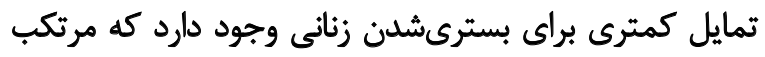
خود كشى شدهاند.

در يُروهش حاضر تلاش شده است با در كنار هم قراردادن

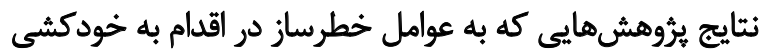

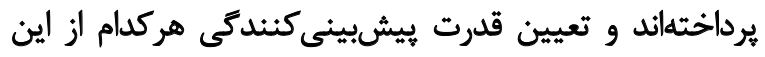

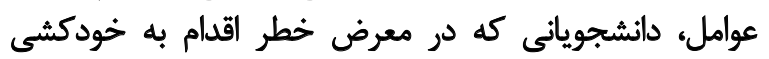

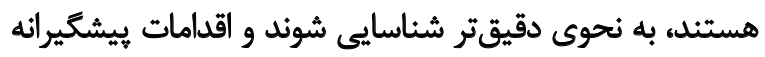

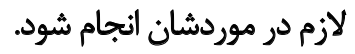
ووشى

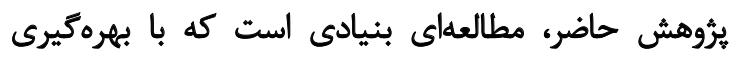

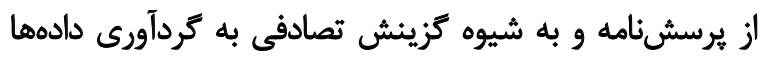

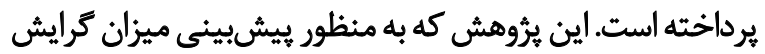

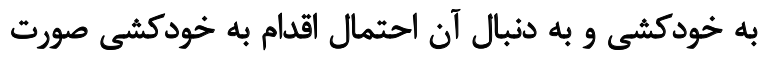

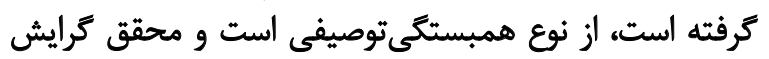

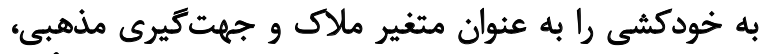

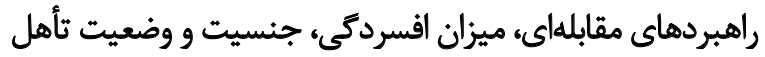

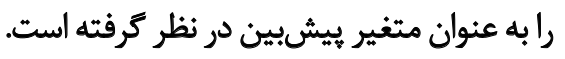

جامعه يروهش شامل تمام دانشجويان مشغول به تحصيل در

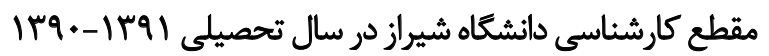

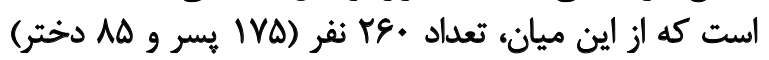

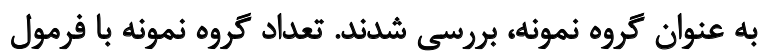

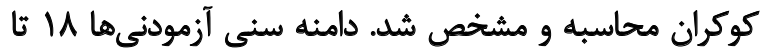

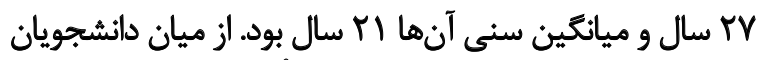

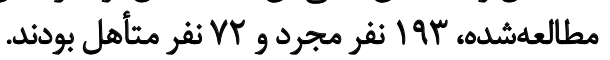
روش نمونه

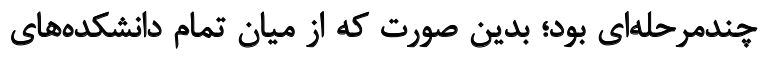

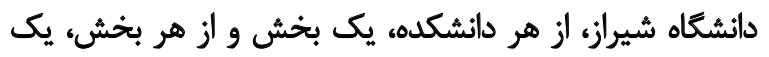

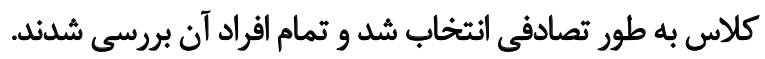

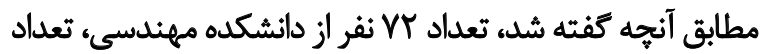
r r نفر از دانشكده كشاورزى، تعداد

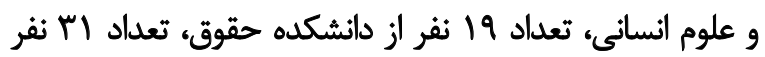

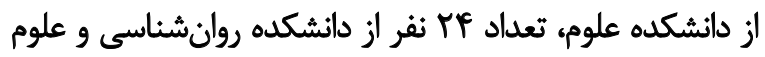

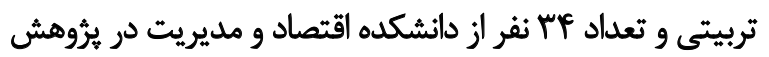
حاضر مطالعه شدند.

11) معيارهاى ورود به مطالعه شامل دانشجويان بزركسال

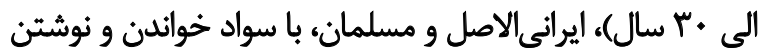

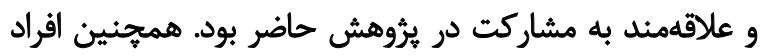

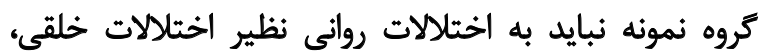


جدول ا. ويرُّى هاى جمعيثشئاختى كروه نمونه

\begin{tabular}{|c|c|c|}
\hline درصد تراكمى & فراوائى (درصد) & يروه نمونه \\
\hline$M T / S$ & $\Lambda \Delta(M T / M)$ & لذختر \\
\hline $1 .$. & $\operatorname{IVA}(\mathscr{N V / T})$ & يسر \\
\hline$n / T$ & $19 \%(M / N)$ & هجرد \\
\hline $1 .$. & $V(T \& / V)$ & مثأهل \\
\hline- & $r \varepsilon+(1+e)$ & كل \\
\hline
\end{tabular}

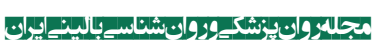

اين مقياس را در سال 1979 بك طراحي كرد و به عنوان

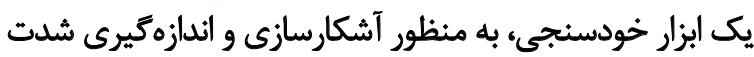

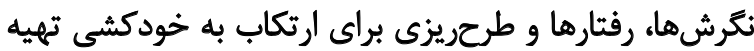

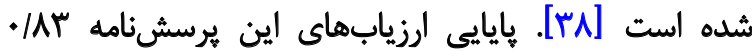

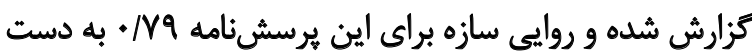

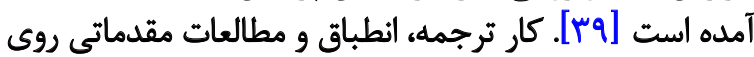

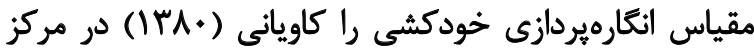

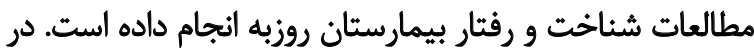

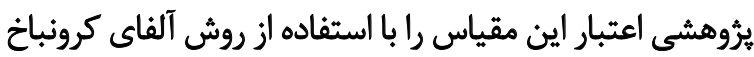

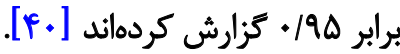

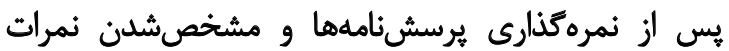

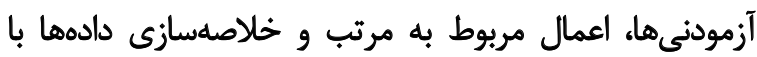

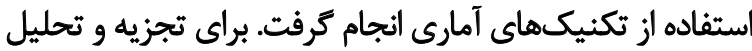

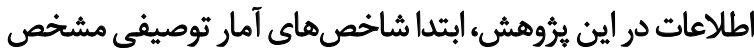

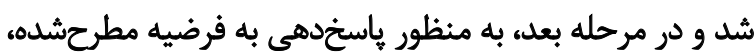

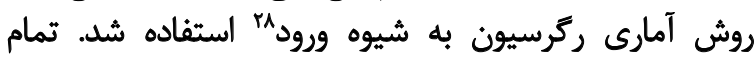

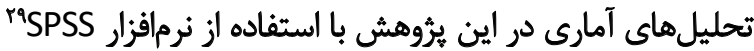

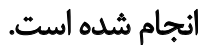

ياقتهها

جنان كه بيشتر بدان اشاره شد، هدف ثروهش حاضر، بررسى

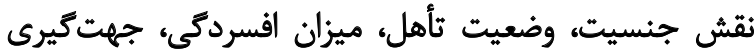

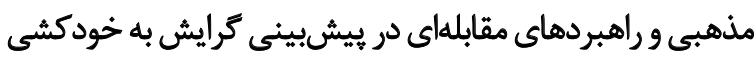

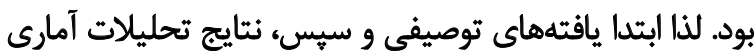
كه به منظور بررسى فرضيات ايثروهش صورت كرفته است، مطرح

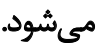

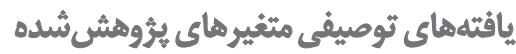

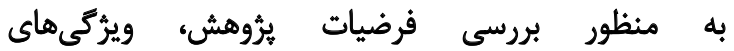
جمعيتشناختى، اطلاعات توصيفى، ميانگين، انحراف معيار،

28. Etnter

29. Statistical Package for the Social Sciences
يرسشنامه شيوهایى مقابله كارور و شيرر (شكل كوتاه) (1919)

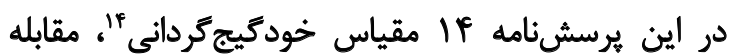

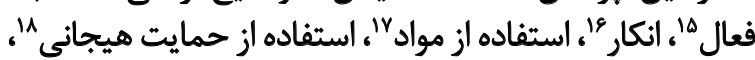

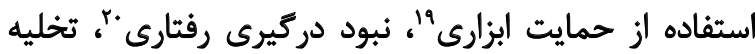

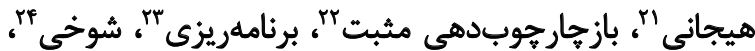

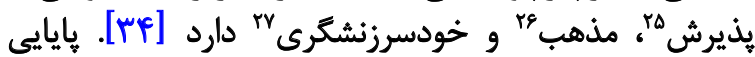

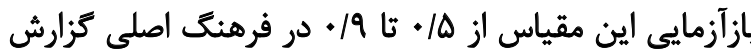

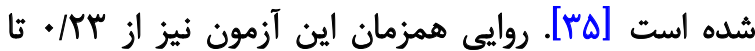

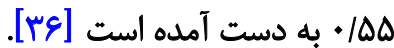
در ايران اين جكليست را براى اولينبار ذوالفقارى و وجاي

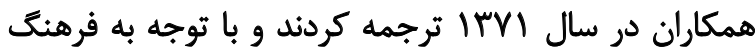

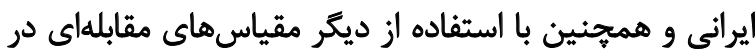

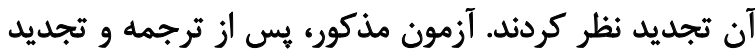

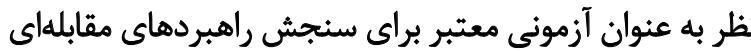

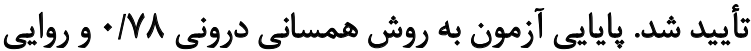

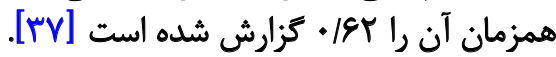
مقياس أنكارهايردازى خودكشى بك

14. Self-distraction

15. Active coping

16. Denial

17. Substance use

18. Use of emotional support

19. Use of instrumental support

20. Behavioral disengagement

21. Venting

22. Positive reframing

23. Planning

24. Humor

25. Acceptance

26. Religion

27. Self-blame 
جدول r. آمار توصيفى مريوط به متغيرهاي بررسىشده (حجم نموئه: •ع))

\begin{tabular}{|c|c|c|c|c|}
\hline انحراف استاندارد & ميانكين & بيشينه & كمينه & متغير بررسيى شده \\
\hline $1 / 41$ & $f / \Delta q$ & V/EV & $r$ & سبك مسئلهمدار \\
\hline $1 / 19$ & $r / T r$ & S/AN & r & سبك ناكارآمد \\
\hline $1 / M$ & $F / r$. & $\checkmark$ & r & سبك هيجان مدار \\
\hline $1.1 \cdot r$ & $\mid r / \Delta T$ & ev & - & ميزان اقسردكى \\
\hline$q / \pi$ & $r \cdot 1 \cdot r$ & ro & 1 & جهت كيرى مذهبي درونى \\
\hline$\Delta / / r$ & $1.10 \mathrm{~V}$ & tr & - & جهت كيرى مذهبي بيرونى \\
\hline$\Delta / \cdot V$ & $r / q)$ & re & - & خطر خودكشى \\
\hline 1/ז & $r / \Delta)$ & $\Lambda$ & $r$ & خودثوجه كردائى \\
\hline$V / N$ & $P / A V$ & $\wedge$ & $r$ & مقابله فعال \\
\hline V & س & $\wedge$ & $r$ & انكار \\
\hline $1 / \% \Delta$ & $r / Q$ & $\wedge$ & 1 & استفاده ازز مواد \\
\hline $1 / \pi V$ & f/Rr & $\Lambda$ & $r$ & استفاده از حمايت هيجانى \\
\hline I/To & $\Gamma / \Lambda \Delta$ & $\checkmark$ & $r$ & استفاده از حمايت ابزارى \\
\hline $1 / 6$ & mptet & $\wedge$ & $r$ & تندانشن دركيرى رفتنارى \\
\hline $1 / 9$. & M/FA & $\wedge$ & $r$ & تخليه هيجاني \\
\hline $1 / M A$ & P/FA & $\Lambda$ & $r$ & بازهارجوبدهى مثبت \\
\hline $1 / v$ & $\Delta / \cdot F$ & 1 & $r$ & برئامهريزى \\
\hline $1 / .8$ & $r / \cdot \varphi$ & $\checkmark$ & r & 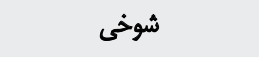 \\
\hline$|/ F|$ & $F / F \Delta$ & $\wedge$ & $r$ & يذيرش \\
\hline$r / \omega$ & $f(\lambda)$ & $\wedge$ & r & مذهب \\
\hline V/IV & m/f. & $\checkmark$ & $r$ & خودسرزنشكرى \\
\hline
\end{tabular}

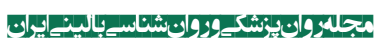

تخليه هيجانى

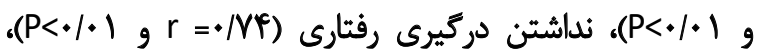

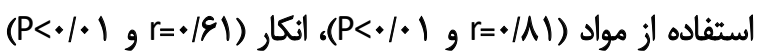
و خودتوجه

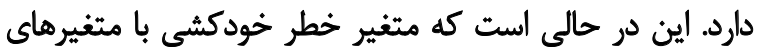

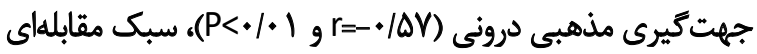
هيجان مدار (Pf| •

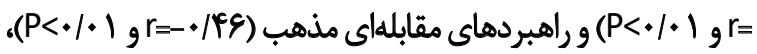
يذيرش

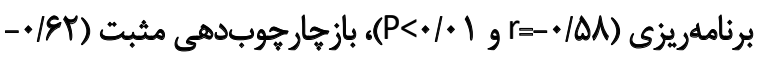

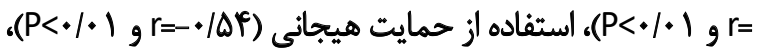

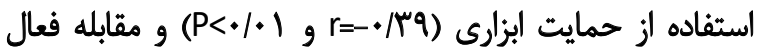

حداقل و حداكثر نمرات، مطالعه شد كه نتايج آن در جداول شماره او و آمده است. ماتِ يس هابس

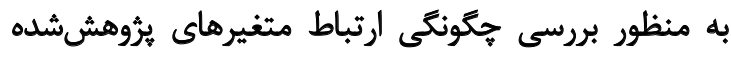

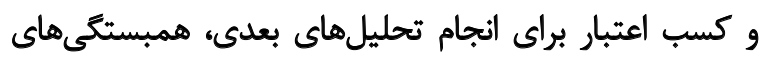

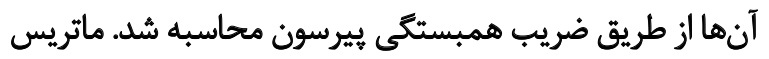

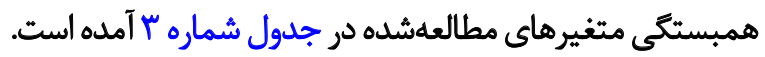

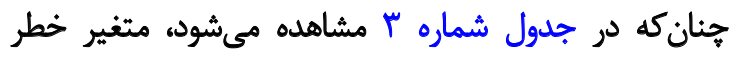

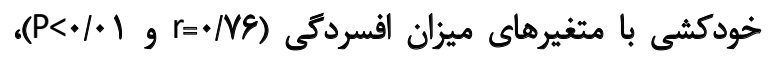

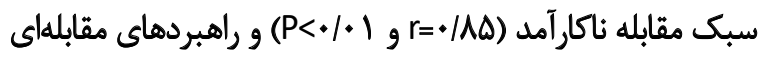


جدول ؟. ماتريس همبستكى متغيرهاي بروسى شده

\begin{tabular}{|c|c|c|c|c|c|c|c|}
\hline V & 7 & $\bullet$ & $\varepsilon$ & $r$ & $r$ & 1 & \\
\hline & & & & & & 1 & ا (. خودتوجه كردانى \\
\hline & & & & & 1 &.$- / N \cdot *$ & ك. يقابله فعال \\
\hline & & & & 1 & $-* / \mathrm{VI}$ & $\cdot N^{*}$ & س. أنكار \\
\hline & & & 1 & $.100^{\circ}$ & -.188 & $.19 V^{*}$ & †. أسثفاده از مواد \\
\hline & & 1 & $-* / 4 r^{2}$ &.$- / / r$ & $\cdot 1 \mathrm{~A}^{*}$ &.$- / r \Delta^{*}$ & هـ استفاده از حمايت هيجانى \\
\hline & 1 &.$/ / r^{*}$ & $-+/ \Delta 0^{*}$ &.$-|\Delta|^{\prime \prime}$ & $\cdot N^{*}$ & $-. / \Delta)^{\prime \prime}$ & ع استفاده از حمايت ابزارى \\
\hline 1 & $-+109^{\circ}$ & $-+/ 4 \pi$ & $\cdot M=*$ & $+/ *^{*}$ & $-+M I^{*}$ & $+/ *^{*}$ & V. نداشتن دركيرى رفتارى \\
\hline$+/ \mathrm{YA}$ & $-+/ \Delta 9^{\circ}$ & $-. / 4)^{*}$ & $\cdot / q^{*}$ & $.18 \Delta^{\circ}$ & $-+/ V \bullet *$ & $\cdot / M^{*}$ & ᄉ نتخليه هيجانى \\
\hline$-\cdot N^{*}$ &.$/ \% q^{*}$ & $+/ \Delta \Delta^{\circ}$ & $-+|8|^{*}$ & $-.18 *^{*}$ & $.1 \Delta F^{*}$ & -.194 & ه. بازجارجوبدهى هثبت \\
\hline$-.19 e^{\circ}$ & . $M e^{e *}$ & $1 \cdot 1$ & $-.1 \Delta \mathrm{A}^{*}$ & $-.10 A^{\circ}$ &.$/ 1 \Delta^{\circ}$ &.$- / e r^{*}$ & ا. برثامهريزى \\
\hline.$- \mid \Delta)^{*}$ &.$/ 1 \Delta^{*}$ &.$/ M E *$ &.$- / p i$ &.$- / e r$ &.$/ \Delta f^{*}$ &.$- / P T^{*}$ & 11. شوخى \\
\hline$-\cdot M T^{*}$ &.$/ p T^{*}$ & $.100^{\circ}$ &.$-|8|^{*}$ & $-.189 *$ & $.1 \Delta \Delta^{*}$ & -.18 & rا. يُّيرش \\
\hline.$- \mid(1)^{*}$ & $\cdot / M *$ & $\cdot / r V^{*}$ & $-\bullet / P \Lambda^{*}$ & $-A N T^{*}$ & $\cdot / r^{*}$ &.$- / p i^{*}$ & "ال. ملهبب \\
\hline$+/ N T^{*}$ & $-* / \pi$ &.$- / M m^{*}$ & $\cdot / r r^{*}$ &.$/ 4 T^{*}$ &.$- / \mu \phi$ &.$/ P T^{*}$ & 11. خُودسرزنشكرىى \\
\hline$-\cdot M^{*}$ & $\cdot 1 e^{*}$ &.$/ 10^{\circ}$ & $-+/ \notin \Delta^{*}$ & $-.18 V^{*}$ & .194 & $-.18 \mathrm{~A}^{*}$ & ها . سبك مقابله مسئلهملار \\
\hline$\cdot / M^{*}$ & $-+/ 9 F^{*}$ & $-+/ 4 a^{*}$ & $.1110^{\circ}$ &.$|A|^{\circ}$ & $-+/ 19^{*}$ & $\cdot \mathbb{N}^{\circ}$ & عا. سبك مقابله ناكارآمد \\
\hline$-+M^{c *}$ &.$/ 40^{*}$ & $+18 \mathrm{~N}^{*}$ & $-+188^{*}$ & $-+180^{\circ}$ &.$/ \Delta \varepsilon^{*}$ & $-.18 r^{*}$ & V. سبك مقابله هيجانمدار \\
\hline +/Ar" & $-+/ 9 F^{*}$ &.$- / 49^{\circ}$ & $\cdot / V \Lambda^{*}$ & $.198^{\circ}$ & $-+/ V \Delta^{*}$ &.$/ V 8^{*}$ & 11. ميزان افسردتى \\
\hline.$- / \Delta F^{*}$ & 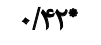 & $\cdot|r| *$ & $-+/ \Delta \mathrm{A}^{*}$ &.$- / 4 T$ &.$(\Delta)^{*}$ & $-\cdot(\Delta)^{*}$ & 19. جهت كيرى مذهبى درونى \\
\hline$-* 1.8$ & $.1 . r$ &.- .1 .1 &.- .111 & $-\infty 1+\infty$ & .1 .0 & $-. / 1 \%+*$ & •r. جهت Sيرى هذهبى بيرونى \\
\hline$\cdot / N^{*}$ & $-+/ \Delta f^{*}$ &.$- / \mu q^{*}$ & $\cdot(A)^{*}$ &.$|8|^{\circ}$ &.$- / 8 T^{*}$ & $\cdot / M *$ & ا. خططر خودكشى \\
\hline \multirow[t]{9}{*}{$1 \varepsilon$} & ir & ir & 11 & $1+$ & 9 & $\Lambda$ & \\
\hline & & & & & & & | أخودتوجلهر دانَى \\
\hline & & & & & & & با مقابله فعال \\
\hline & & & & & & & "ائار \\
\hline & & & & & & & f. إستفاده از مواد \\
\hline & & & & & & & هـ استفاده از حمايت هيجانى \\
\hline & & & & & & & و استفاده از حمايث ابزارى \\
\hline & & & & & & & V. ندائن دركيرى رفتارى \\
\hline & & & & & & 1 & 1 تخطليه هيجاني \\
\hline
\end{tabular}




\begin{tabular}{|c|c|c|c|c|c|c|c|}
\hline $1 \varepsilon$ & it & it & 11 & $1+$ & 9 & $\wedge$ & \\
\hline & & & & & 1 & $-+189 * *$ & 9. بازجارجيوبدهى مثبت \\
\hline & & & & 1 & 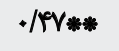 & $-\cdot 18 \cdot$ 䄅粪 & . ا. برنامهريزى \\
\hline & & & 1 &.$/ P V *$ & -/ $199 *$ & $-\bullet / p q * *$ & 11. شوخى \\
\hline & & 1 & $-(01 * *$ &.$/ A f *$ & -No** & $-\cdot 18 \wedge * *$ & rا. يذيرش \\
\hline & 1 & $\cdot \mid \Delta \rho *$ &.$M I * *$ & $. / M \mid * *$ & $.18 T * *$ & $-\cdot 10 \cdot * *$ & "ا. مذهب \\
\hline 1 & $\%$ & $-. / 4 \mid * *$ & $-+/ 4 \mid * *$ & $-\cdot / 4 \mid * *$ & $-\pi / 4 r$ *** &.$/ 4 \Delta *$ & 1f. خودسرزنشكرى \\
\hline$-\odot / f f+*$ & $\cdot / K \xi_{*}^{* * *}$ & -/Ar & $\cdot / \Delta r^{*} *$ &.$/ 94 *$ & $\cdot / \Delta r$ w & $-.189 * *$ & 1ه . سبك مقابله مسئلهمدار \\
\hline$+18 \cdot *$ & 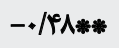 & $-\cdot / V \& * *$ & $--/ \Delta F$ & $-.189 * *$ & - - No &.$/ 91 *$ & عا. سبك مقابله ناكارآمد \\
\hline$-\cdot / \Psi \Delta *$ & $\cdot / 9^{*} *$ & $\cdot / \mathcal{N} *$ &.$|8| * *$ &.$/ 100 *$ & $\cdot / 19 * *$ & $-+/ \mathrm{Y}) * *$ & IV. سبك مقابله هيجان مدار \\
\hline$\cdot / \Delta Y$ & $-/ \times 99$ & $-\cdot / N^{2}$ & $--/ \Delta A$ * & $-\cdot / N \cdot$ 紫䅈 & 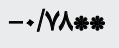 & . $\mid$ A| & 11. هيزان افسردكى \\
\hline.$- / 1$ & •/AY䄅 & . $/ \Delta T^{*}$ & ・/゙人䄅 &.$/ 47$. & $\cdot|8|$ 䄅絭 & -.187 管 & 19. جهت كيرى مذهبي دروئى \\
\hline .1 .0 & •/MP絭䊑 & .1 .9 & .1 .0 & $-.1 \cdot 1$ &.$/ 10$ * & $-\infty 1$ & • ץ. جهت كيرى مذهبى بيرونى \\
\hline$\cdot / \Re r$ & $-\pi / 48$ & $--109 * *$ & 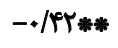 & $--/ \mathrm{AA}$ ***** & - IEr & $\cdot|A|$ & آ. خطر خودكشى \\
\hline \multirow[t]{19}{*}{ M } & $r+$ & 19 & M & iv & 17 & 10 & \\
\hline & & & & & & & ا . خودتوجه كردانى \\
\hline & & & & & & & r. مقابله فعال \\
\hline & & & & & & & 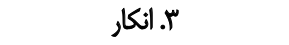 \\
\hline & & & & & & & f. أستفاده از مواد \\
\hline & & & & & & & هـ استفاده از حمايت هيجانى \\
\hline & & & & & & & ع استفاده از حمايت ابزارى \\
\hline & & & & & & & Y. نداشتن دركيرى رقتارى \\
\hline & & & & & & & A إخليه هيجانى \\
\hline & & & & & & & ج. بازئجاريوبدهى مثبت \\
\hline & & & & & & & ا. برثاملريزى \\
\hline & & & & & & & ||l. شوخى \\
\hline & & & & & & & rا. ئذيرش \\
\hline & & & & & & & "اله مذهب \\
\hline & & & & & & & أ خودسرزنشكرى \\
\hline & & & & & & 1 & ها. سبك مقابله مسئلهمدار \\
\hline & & & & & 1 & $-+/ \mathrm{YA}$ & 18. سبك مقابله ناكارآمد \\
\hline & & & & 1 & $-{ }^{+} / V q_{\text {粪 }}$ & $\cdot / \Delta H^{*}$ & IV. سبك مقابله هيجانمدار \\
\hline & & & 1 & - - VA** & -119** & $-\bullet / V \&$ & 11. ميزان افسردكى \\
\hline
\end{tabular}




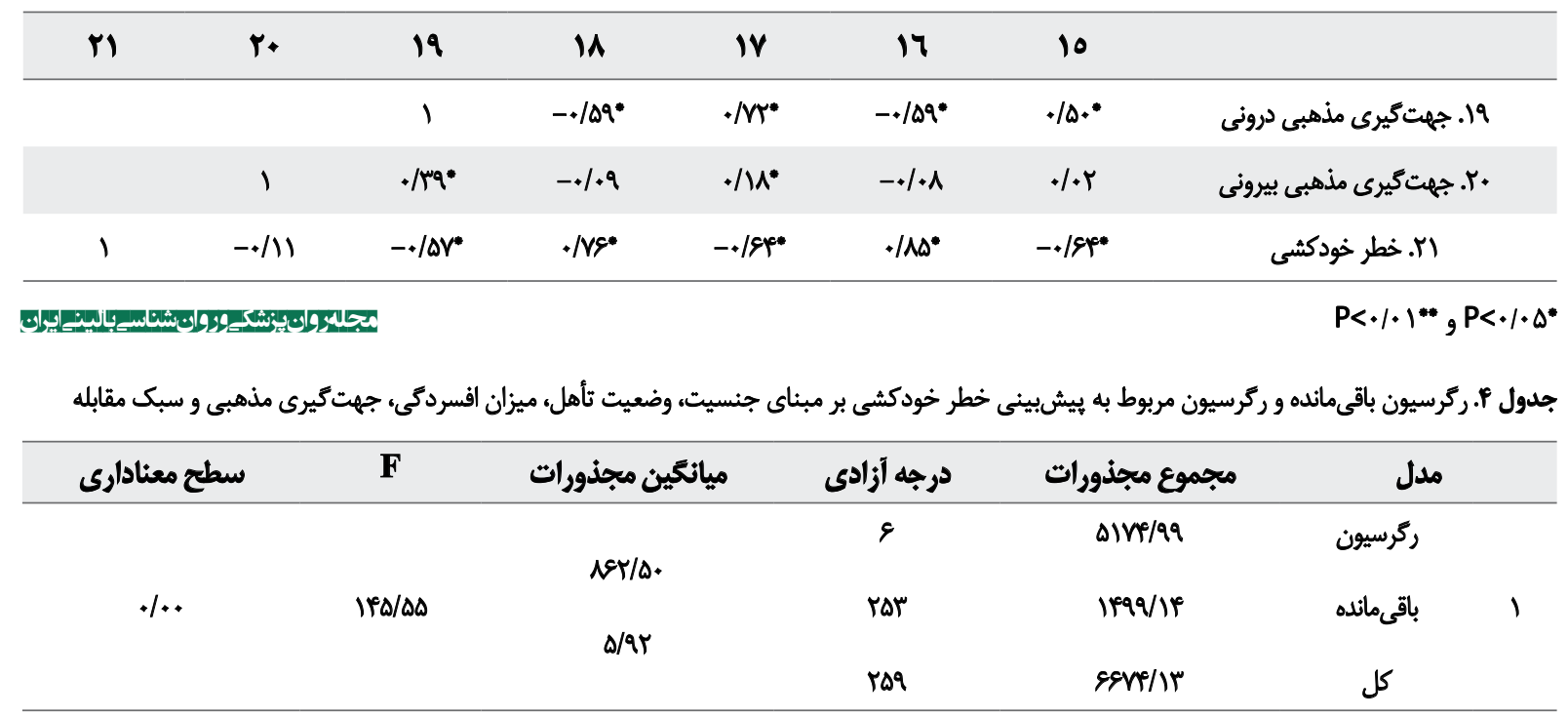

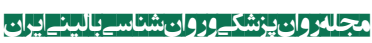

دركيرى رفتارى و جنسيت مى توانيد ل لدرصد از واريانس خطر

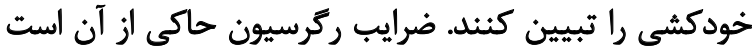

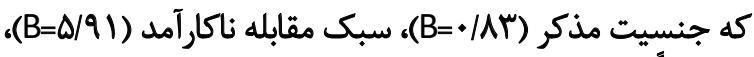

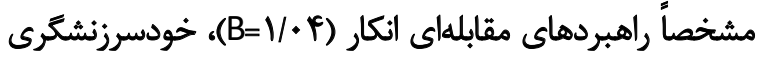
(B=॰|Al)

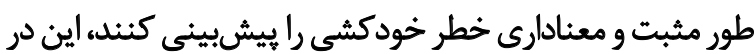

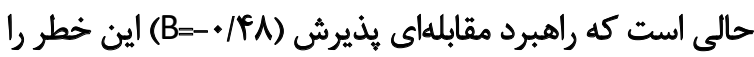

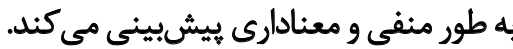

بحث

هدف بروهش حاضر، بررسى سهم جنسيت، وضعيت تأهل،

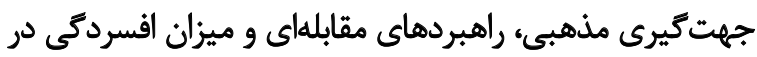

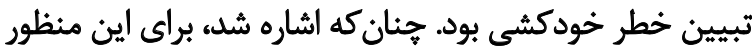
از روش ركرسيون به شيوه ورود استفاده شد. نتايج نشان دان داد
به طور منفى و معنادارى رابطه دارد. براى بررسى فرضيات مطرحشده در يُوهش حاضر، خطر

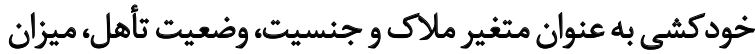

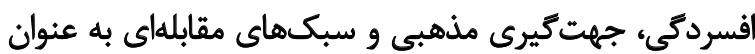

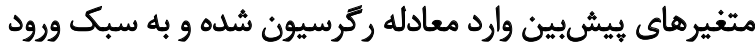

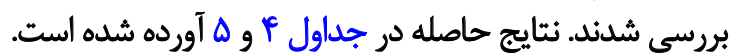
جدول شماره F نشان مى دهد مقدار F براى بررسى توانايى

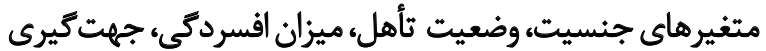

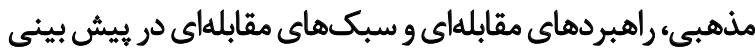

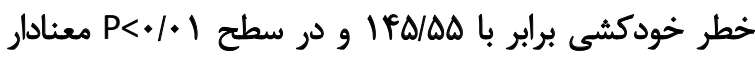
است. خطر خو

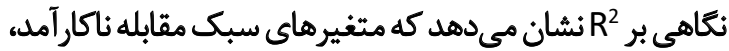

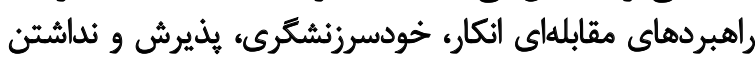
جدول هـ ضرايب ركرسيون مربوط به بيشبينى خطر خودكشى بر مبناى جنسيت، وضعيت تأهل، ميزان افسردىى، جهتكيرى مذهبي، سبكهاى مقابله و

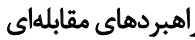

\begin{tabular}{|c|c|c|c|c|c|c|}
\hline سطح معنادارى & $\mathbf{t}$ & $\mathbf{R}^{2}$ & $\mathbf{R}$ & ضرايب رتمرسيون & مثغير ييشبين & مدل \\
\hline$\%$ & $-V / r q$ & & & $-1 \cdot / \pi$ & ضريب ثابت & 1 \\
\hline$\%$ & $11 / M^{e}$ & & & $\Delta / 91$ & سبك مقابله ناكارآمد & \\
\hline$\%$ & $H / A$. & & & $1 / \cdot 5$ & انكار & \\
\hline$\%$ & e/MA &.$/ \mathrm{NA}$ & $\cdot / M$ & $A V /$. & خودسرزنشكرى & \\
\hline .1 .1 & $-r / 8$. & & & $=. / 21$ & ل بذيرش & \\
\hline.$/ 1$ & t/Rg & & &.$/ A r$ & جنسيت (مذكر) & \\
\hline 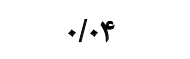 & $r / \cdot 1$ & & &.$/ 4 q$ & نداشتن دركيرى رفتارى & \\
\hline
\end{tabular}


حمايتهاي قابل دسترس، نوع راهبرد مقابلهاي او را مشخص مى كند [FV] به عبارت ديكر، زمانى كه فرد خود رادر موقعيتى نار احت كنينده

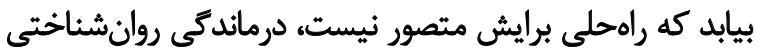

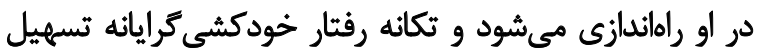

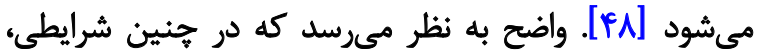

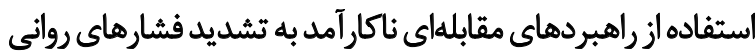

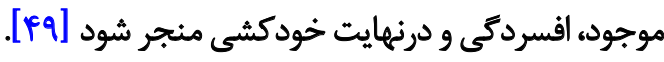

براى تبيين رابطه مثبت و معنادار ميزان افسردكى و افكار

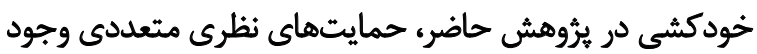

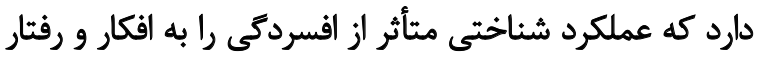

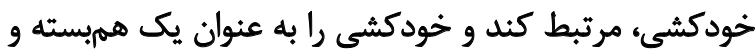

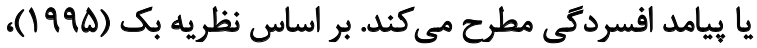

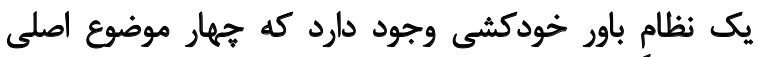

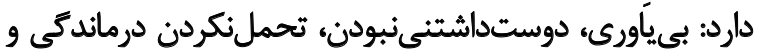

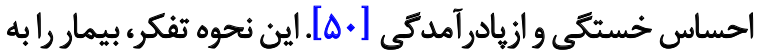

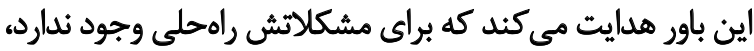

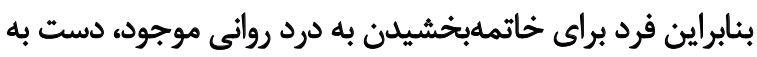
خودكشى مىزند [ [هائ.

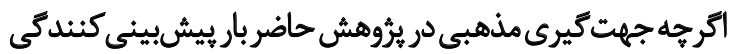

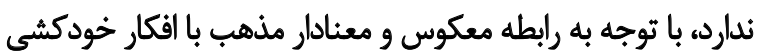
(r=-•/DV)

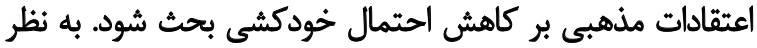

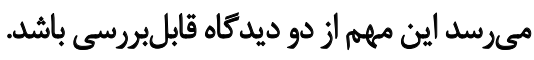

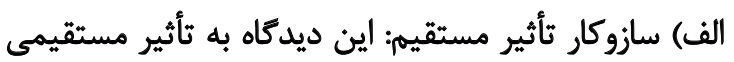

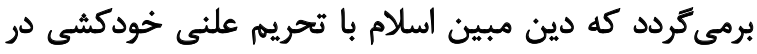

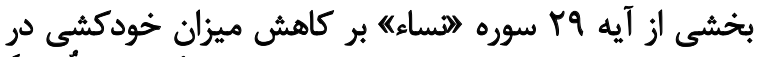

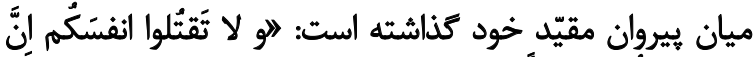

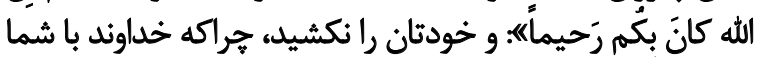

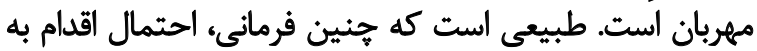

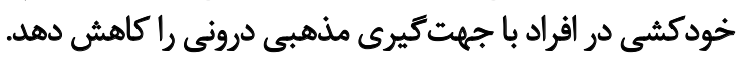

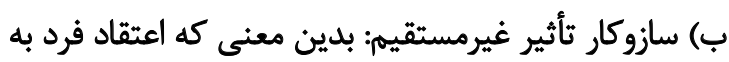

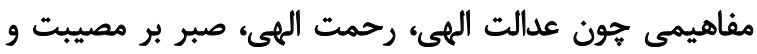

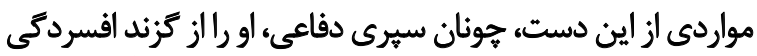

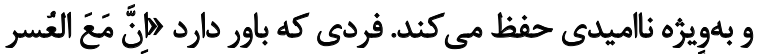

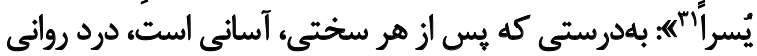

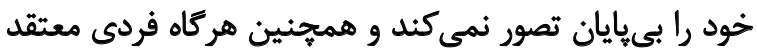

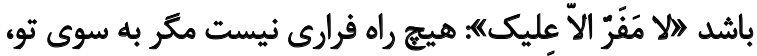

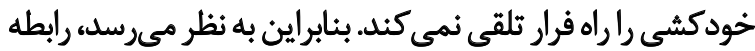

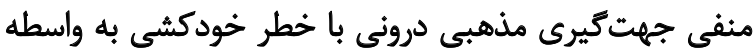

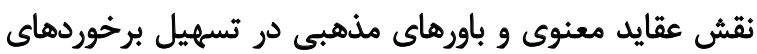

كه جهت كيرى مذهبى درونى، سبكهاي مقابلهاى مسئلهمدار

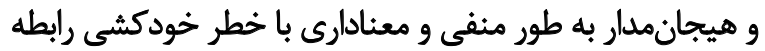

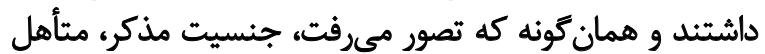

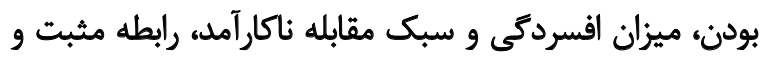

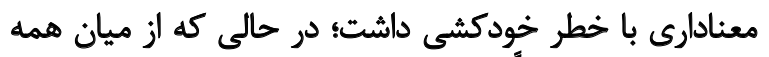

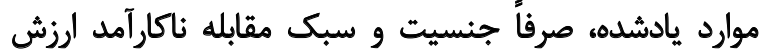
بيشبينى كنيندكى داشت.

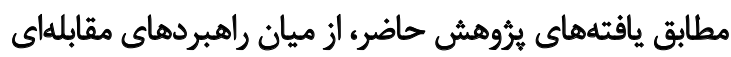

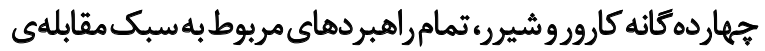

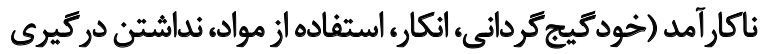

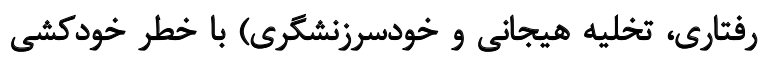

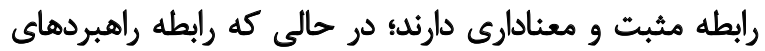

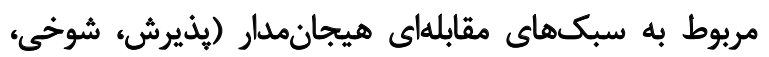

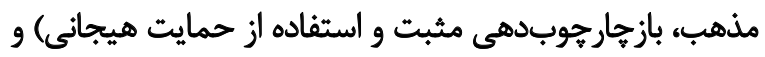

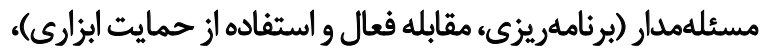

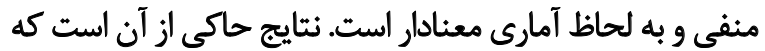

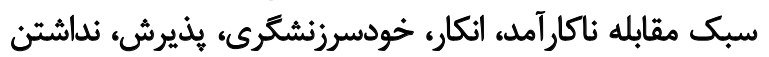

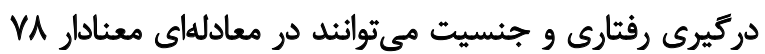
درصد واريانس خطر خودكثى را تبيين كنند.

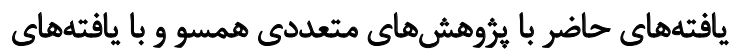

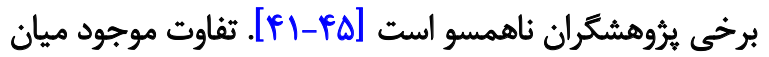

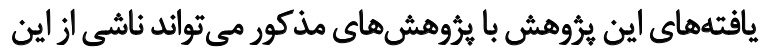

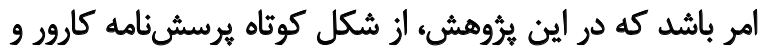

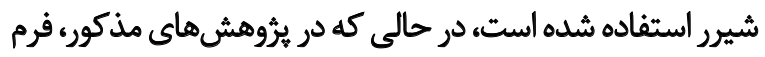

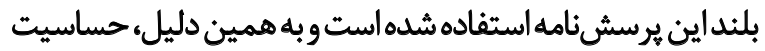

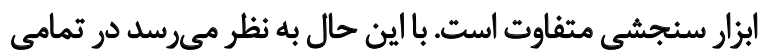

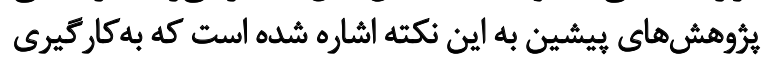

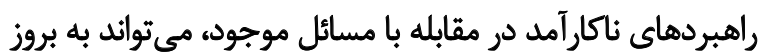

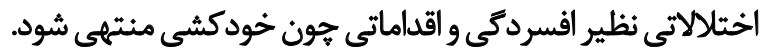

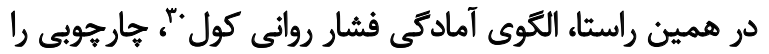
براى درمان افراد خودكشى كننده فراهم كرده است.

بر اساس اين مدل، افرادى كه در توانايى تفكر وآترا مشكل دارند، زمانى

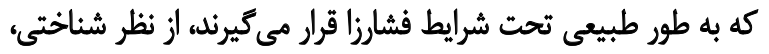

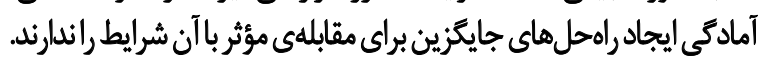

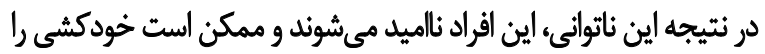

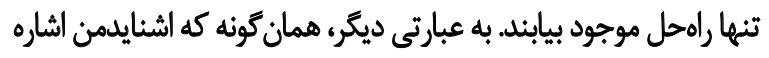

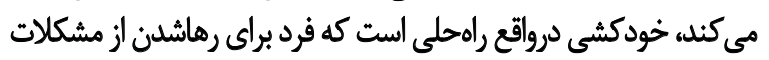

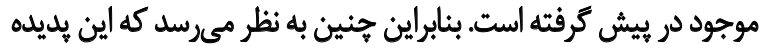

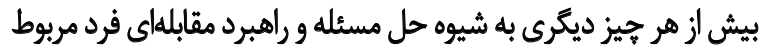

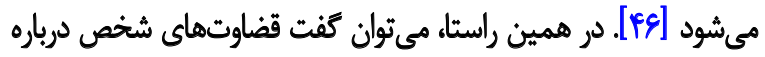

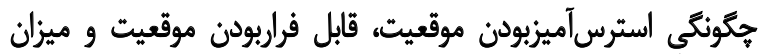


بنابراين انتظار مىرود روانشناسان به اين سازه توجه ويرهاى

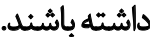

از جمله محدوديتهاى يُروهش حاضر، ميتوان به تعدد

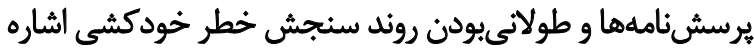

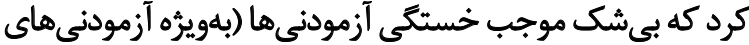

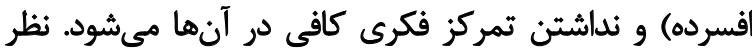

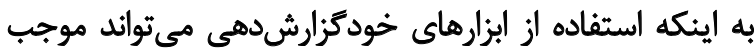
تحريف اطلاعات از سوى آزمودنىها شود، استفاده ازئ إز مصاحبه

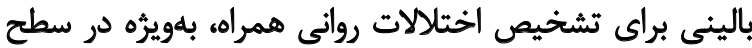

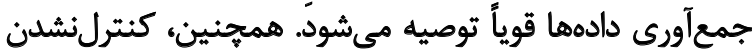

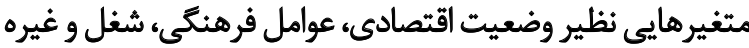

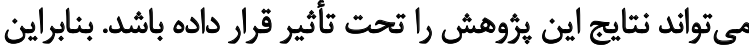

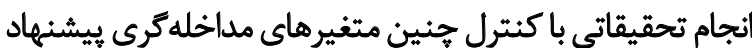

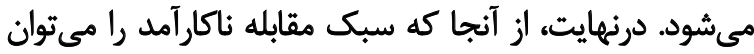

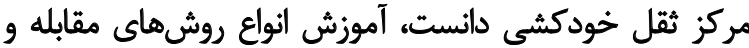

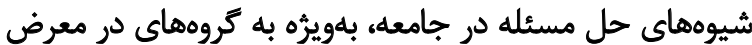

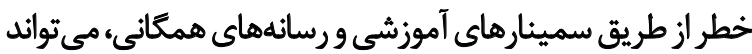
اقدامى مؤثر در در كاهش خطر خودكشى باشد.

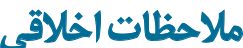

\section{ييروى از اصول الخاق بورهش}

يروتكل ثئوهش حاضر را كميته اخلاقى دانشعاه شيراز تأييد كرده

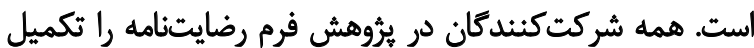

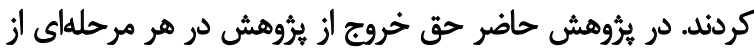

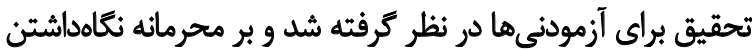
ياسخهاى شركت كنيدكان همواره تاكيد مي كرشد.

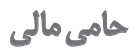

اين يروهش از يايانئامه كارشناسيارشد نويسنده اول در رشته

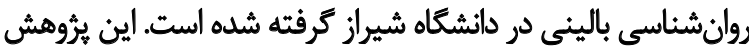

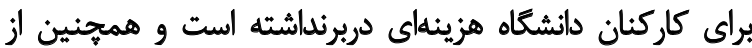
هيجكدام از منابع مالى دانشعاه شيراز استفاده نشده است.

مشاركتثويسندكان

طراحى يُروهش: ياسمن عماد و حبيب هاديانفرد. جمعآورى

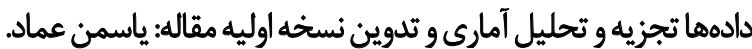

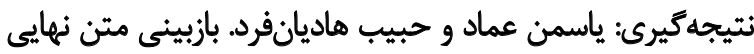
مقاله و بازبينى نثًارشى و علمى حبيب هادي هاديانفرد.

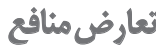

بنابر اظهار نهويسندكان، مقاله تعارض منافع ندارد.
سازشى و يرهيز از نوميدى قابل توجيه باشد.

مطابق يافتهها در اين يُؤهش، جنسيت مذكر ميتواند

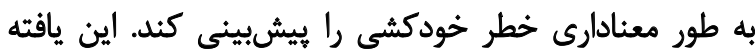

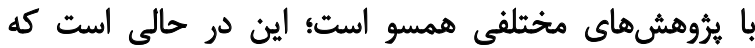

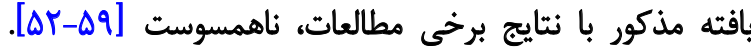

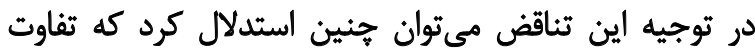

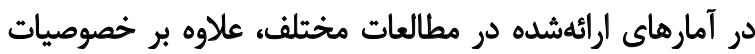

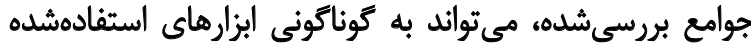

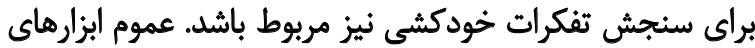

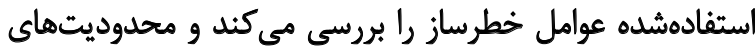
قابل توجهى در ارزيابى شدت انتارهيردازي خودكشى مادى دارد.

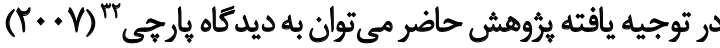

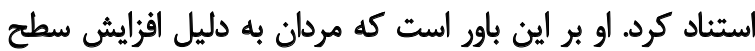

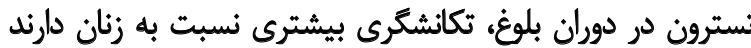

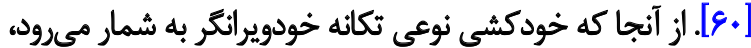

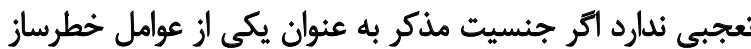

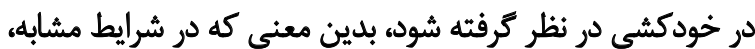

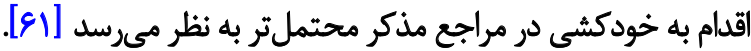

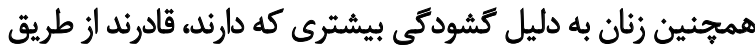

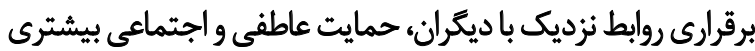

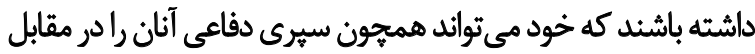

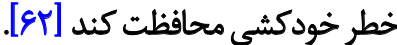

در تبيين ديكر يافتهها، مي توان به طور ضمنى اينكونه استنباط

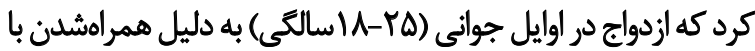

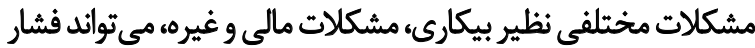

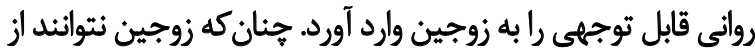

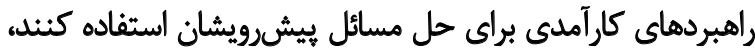

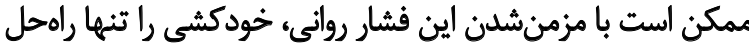

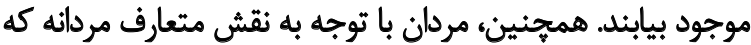

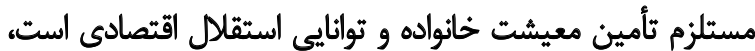

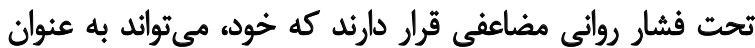

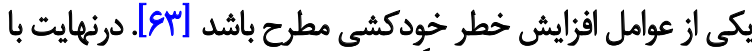

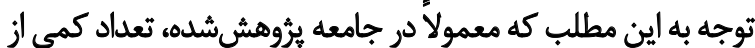

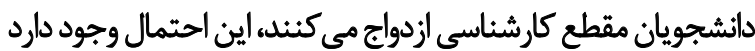
كه تفاوتهاى آمارى موجود، مربوط بارئه اين مسئله باشد.

\section{تئيجهيرى}

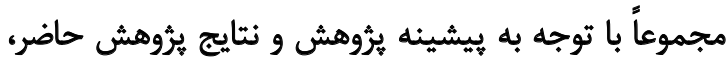

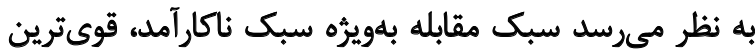

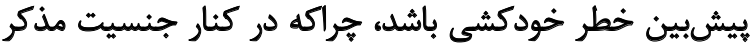
مى يواند VA درصد واريانس خطر خودئ خودكشى را تبيين كند.

32. Parchi 


\section{References}

[1] Zare H, Nazer M, Sayyadi A. [Frequency of mental disorders and factors related to suicide in patients referring to emergency ward of Ali Ebne Abitaleb Hospital of Rafsanjan in 2007 (Persian)]. Journal of Rafsanjan University of Medical Sciences. 2010; 9(3):221-32.

[2] Lester D. Suicide prevention: Dying and bereavement. Abingdon-on-Thames: Routledge; 2001.

[3] Pereira EJ, Kroner DG, Holden RR, Flamenbaum R. A comparative study of attitudes toward suicide among Swedish and Persian high school students. Psychological Research. 2010; 49(8):912-7. [DOI:10.1016/j.paid.2010.07.029]

[4] Khajehmougahi N, Behrouzian F, Ghanavati F. [The investigation of relationship between hopelessness and suicide among mood disorders patients (Persian)]. Journal of Medical Science. 2009; 8:407-13.

[5] Mahmody S, sSalhnegad G, Nazaryan S, Yaghobi M. [A comparison study of depression between hemodialysis patients and renal transplant recipients (Persian)]. Iranian Journal of Nursing Research. 2010; 5(18):73-80.

[6] Oquendo M, Brent D, Birmaher B, Greenhill L, Kolko D, Stanley B, et al. Posttraumatic stress disorder comorbid with major depression: Factors mediating the association with suicidal behavior. American Journal of Psychiatry. 2005; 162(3):560-6. [DOI:10.1176/appi.ajp.162.3.560] [PMID]

[7] Troister T, Holden RR. Comparing psychache, depression, and hopelessness in their associations with suicidality: Atest of Shneidman's theory of suicide. Personality and Individual Difference. 2010; 49:689-93. [DOI:10.1016/j.paid.2010.06.006]

[8] Zacharkis CA, Madianson MG. Suicide in Greece 1980-1995: Patterns and social factors. Journal of Psychiatry Epidemiolog. 1998; 33(10):471-6. [DOI:10.1007/s001270050081]

[9] Hakery J, Sadeghy KhA. The role of life events and coping styles in relapse of Post-Traumatic Stress Disorder in a sample of war injured (Kermanshah). Journal of Military Medicine. 2003; 5(2):111-6.

[10] Besharat MA, Pourang P, Pourtabai AS, Pournaghsh Tehrani, $\mathrm{S}$. [The relationship between coping styles and psychological adaptation in the recovery process: Patients with coronary heart disease (Persian)]. Tehran University Medical Journal. 2008; 66(8):573-9.

[11] Shamsi Khani S, Rahgoo A, Fallahi Khoshknab M, Rahgozar M. [Effects of problem solving training on coping skills of suicidal clients (Persian)]. Iranian Journal of Nursing Research. 2007; 1(3):31-9.

[12] Atarodi M. [The relationship between documentary styles, coping strategies and mental health in high school students in Shiraz (Persian)] [PhD dissertation]. Shiraz: Shiraz University; 2012.

[13] Alport GW. Religious context of prejudice. Journal for the Scientific Study of Religion. 1966; 5(12):7-21. [DOI:10.2307/1384172]

[14] Bahrami Ehsan S, Tamanaefar H, Bahrami Ehsan Z. [The relationship between dimensions of religious orientation, mental health and psychological disorders (Persian)]. Iranian Journal of Psychologists. 2005; 2(5):35-42.
[15] Hosenchari M, Namian S. [The prediction of students' educational negligence based on core control and religious beliefs (Persian)]. Journal of Educational Psychology Studies. 2011; 8(14):99-126.

[16] Janbozorgi, M. [Religious orientation and mental health (Persian)]. Research in Medicine. 2009; 31(4):345-50.

[17] Rezaeian M, Vazirinejad R, Tabatabie S, Salem Z, Esmaili A. [Suicide in Islamic faith (Persian)]. Journal of Rafsanjan University of Medical Sciences. 2007; 6(4):15-30.

[18] Ghalambor A, Zarei J, Pipelzadeh MH. Assessment of personal and social characteristics as risk factors in self-inflicted burns (Persian)]. Journal of Medical Science. 2010; 9(3):233-45.

[19] Shamsalizadeh N, Afkhamzadeh A, Mohsenpour B, Salehian B. Suicide attempt and related factors in Kurdistan province. Scientific Journal of Kurdistan University of Medical Sciences. 2010; 15(1):79-86

[20] Anbari M, Bahrami A. [Study of the impact of poverty and violence on suicide rate in Iran: Case study (Persian)]. Iranian Journal of Social Problems. 2010; 1(2):1-30.

[21] Memari A, Ramim T, Amirmoradi F, Khosravi K, Godarzi Z. Causes of suicide in married women (Persian)]. Hayat. 2006; 12 (1):47-53.

[22] Barker-Collo SL. Adult reports of child and adult attributions of blame for childhood sexual abuse: Predicting adult adjustment and suicidal behaviors in females. Child Abuse \& Neglect. 2001; 25(10):1329-41. [DOI:10.1016/S0145-2134(01)00278-2]

[23] Tavallaii SA, Ghanei M, Assari SH, Lorgarde Dezfuli Nezhad M, Habibi M. [Risk factors correlated to suicide in deceased Iranian veterans (Persian)]. Journal of Military Medicine. 2006; 8(2):143-8.

[24] Keshmiri M. [Comparison of lifestyle and coping strategies of teachers of exceptional and normal education (Persian)]. Journal of Exceptional Education. 2008; 98(1):3-21.

[25] Nezamipour E, Abdolmanafi A, Besharat MA. [Comparison of coping strategies and problem-solving styles in depressed and non-depressed students (Persian)]. Shenakht Journal of Psychology and Psychiatry. 2015; 2(2):59-69.

[26] Beck AT, Steer RA, Brown GK. Manual for the Beck Depression Inventory-II. San Antonio, TX: The Psychological Corporation; 2000 .

[27] Stefan-Dabson K, Mohammadkhani P, Massah-Choulabi O. Psychometrics characteristic of Beck Depression Inventory-II in patients with magor depressive disorder. Journal of Rehabilitation. 2007; 8(suppl. 29):82-8.

[28] Hasani J, Hadianfard H. Comparison of attentional care in patients with schizophrenia, major depression, and normal people. Journal of Psychology and Education. 2007; 37(1):159-84.

[29] Makarem S, Goodarzi MA, Taghavi SMR, Rahimi CH. [A comparison between performance of patients with schizophrenia, major depression and normal controls in spatio-temporal integration task (Persian)]. Journal of Psychology. 2010; 14 (2):114-30.

[30] Pedram M, Mohammadi M, Naziri GH, Aeinparast, N. Effectiveness of cognitive-behavioral group therapy on the treatment of anxiety and depression disorders and on raising hope in women with breast cancer. Quarterly Journal of Women and Society. 2009; $1(4): 61-75$ 
[31] Bayani AS, Goodarzi H, Bayani A. [Investigating the relationship between religious orientation and anxiety and depression in students (Persian)]. Journal of Fundamentals of Mental Health. 2010; 10(4):209-14

[32] Nasabeh SMH. [The role of religion and religious beliefs on mental health (Persian)]. Pazhouhesh Dini. 2006; 14(3):71-94.

[33] Rostami M, Hashemi T, Aliloo MM. [Comparison of personality traits, social support, and religious psychology in suicide attempters and control group (Persian)]. The Journal of Urmia University of Medical Sciences. 2014; 24(7):1016-26.

[34] Carver CS. You want to measure coping but your protocol's too long: Consider the brief cope. International Journal of Behavioral Medicine. 1997; 4(1):92. [DOI:10.1207/s15327558ijbm0401_6] [PMID]

[35] Heibati H. [Studying parental parenting practices and their relationship with stress coping strategies in third grade high school students in Zarghan City (Persian)] [PhD dissertation]. Shiraz: Shiraz University; 2002.

[36] Agha Yousefi AR. [Coping ways to post-traumatic stress disorder and chronic stress symptoms (Persian)]. Journal of Behavioral Sciences. 2010; 4(1):29-34

[37] Nasirzadeh R, Rassolzadeh Tabatabaie K. [Religious beliefs and coping strategies in students (Persian)]. Horizon of Medical Science. 2009; 15(2):36-45.

[38] Rahimi M. [Comparison of cognitive processes in depressed patients with suicidal thoughts and depressed patients without suicidal ideation (Persian)] [PhD dissertation]. Tehran: Tehran University of Medical Science; 1999.

[39] Danitz M. Suicide an unnecessary death. London: Informa Healthcare; 2001.

[40] Anisi J, Fathi Ashtiani A, Salimi SH. [Assessing reliability and validity of Beck Scale for Suicide Ideation (BSSI) among soldiers (Persian)]. Military Medicine. 2004; 7(1):33-7.

[41] Kidd SA, Carroll MR. Coping and suicidality among homeless youth. Journal of Adolescence. 2007; 30(2):283-96. [DOI:10.1016/j. adolescence.2006.03.002] [PMID]

[42] Andover MS, Peter CM, Gibb BE. Self-mutilation and coping strategies in a college sample. Suicide and Life-threatening Behavior. 2007; 37(2):38-43. [DOI:10.1521/suli.2007.37.2.238] [PMID]

[43] Kotler, M. Correlates of suicide and violence risk in an inpatient population: Coping styles and social support. Psychiatry Research. 1993; 47(3):281-90. [DOI:10.1016/0165-1781(93)90085-U]

[44] Jahangir F, Bazrafshan MR, Zangoui A. [Comparison of coping mechanisms used by suicidal attempt patients, and those without suicidal history (Persian)]. Hormozgan Medical Journal. 2009; 13(2):109-13

[45] Chapman A, Specht MW, Cellucci T. Factors associated with suicide attempts in female inmates: The hegemony of hopelessness. Suicide and Life-thereatening Behavior. 2005; 35(5):558-70. [DOI:10.1521/suli.2005.35.5.558] [PMID]

[46] Cole DA. Psychopathology of adolescent suicide: Hopelessness, coping beliefs and depression. Journal of Abnormal Psychology. 1989; 98(3):248-55. [DOI:10.1037/0021-843X.98.3.248] [PMID]
[47] Duberstein PR. Are closed-minded people more open to the idea of killing themselves? Suicide \& Life-threatening Behaviors 2001; 31(1):9-14. [DOI:10.1521/suli.31.1.9.21309] [PMID]

[48] Nierop A, Bratsikas A, Zimmermann R, Ehlert U. Are stressinduced cortisol changes during pregnancy associated with post partum depressive symptoms? Psychometric Medicine. 2006; 68(6):931-7. [DOI:10.1097/01.psy.0000244385.93141.3b] [PMID]

[49] Salehi H, Simbar M, Abolghasemi A, Abadi AR. [A comparison of postpartum depression among low-risk-pregnant women with emotion and problem focused coping strategies (Persian)]. Qom University of Medical Sciences journal. 2013; 7(1):91-96

[50] Rudd MD. Threating suicidal behavior: An effective, time-limited approach. New York: Guilford Press; 2001.

[51] Shneidman E. Suicide as Psychache: A clinical approach to selfdestructive behavior. Northvale. NJ: Jason Aronson; 1993.

[52] Applebay L, Shaw J, Amos T, McDonnell R, Harris C, McCann K. Suicide within 12 Months of Contact with Mental Health Services: National Clinical Survey. British Medical Journal. 1999; 318 1235-9. [DOI:10.1136/bmi.318.7193.1235] [PMID] [PMCID]

[53] Mościcki EK. Epidemiology of suicide behavior. Suicide and Life-threatening Behavior. 1995; 25(1):22-35.

[54] Murphy GE, Wetzel RD. The life-time risk of suicide in alcoholism. Archives Of General Psychiatry. 1990; 47(4):383-92. [DOI:10.1001/archpsyc.1990.01810160083012] [PMID]

[55] Rezaian M, Mohammadi M, Akbari M, Maleki M. [Pattern of suicide in five years recorded cases for Behesht Zahra Organization of Tehran (Persian)].Journal of Research in Behavioural Sciences. 2007; 3(2):5-11.

[56] Yaghobi Y, Shafipour S. [Survey socio-demographic characteristics of adolescents attempted suicide with rice tablet in Razi Hospital in Rasht (Persian)]. The Journal of Nursing and Midwifery Faculties Guilan Medical University. 2009; 17(58):45-50.

[57] Mohammadi S, Izadpanah Sh, Fazeli Mehrabadi A. [The effects of identity styles on suicidal thoughts among university students (Persian)]. Journal of Research in Biological Sciences. 2011; 5(1):61-7.

[58] Mousavi SGh, Malekian A, Keikhaie N. [Relative frequency of suicidal ideation in students of Isfahan universities in 2005 (Persian)]. Hakim Research Journal. 2008; 11(3):55-9.

[59] Rezaian M, Sharifi-rad G. [Self-immolation the most important suicide method in Ilam province (Persian)]. Iranian Journal of Psychiatry and Clinical Psychology. 2006; 12(3):289-90.

[60] Parchi K. Utility of the personality assessment Inventory in assessing suicide risk [MA thesis]. Toledo, Ohio: The University of Toledo; 2007

[61] Pirkis JE, Burgess PM, Meadows GN. Suicidal ideation and suicide attempts as predictors of mental health service use. Journal of Medicine. 2001; 175(10):542-55.

[62] Pettit JW, Joiner TE. Chronic depression: Interpersonal sources, therapeutic solutions. Washington, D.C: American Psychological Association; 2006. [DOI:10.1037/11291-000]

[63] Mohammadzade J. Investigation of prevalence rate of depression symptoms among Ilam University students (Persian)]. Ilam University of Medical Sciences. 2011; 19(2):71-6. 
This Page Intentionally Left Blank 